\title{
BEAT-FREQUENCY MODELS OF KILOHERTZ QPOS
}

\author{
M. Coleman Miller \\ University of Maryland
}

\begin{abstract}
Kilohertz QPO sources are reasonably well-characterized observationally, but many questions remain about the theoretical framework for these sources and the consequent implications of the observations for disk physics, strong gravity, and dense matter. We contrast the predictions and implications of the most extensively studied class of kilohertz QPO models, the beat-frequency models, with those of alternative classes of models. We also discuss the expected impact of new observations of these sources with satellites such as Chandra, XMM, and Constellation-X.
\end{abstract}

KEYWORDS: neutron stars; general relativity; X-ray

\section{INTRODUCTION}

Soon after the launch of the Rossi X-ray Timing Explorer (RXTE) in late 1995, observations with it of neutron star low-mass X-ray binaries revealed kilohertz quasi-periodic brightness oscillations (QPOs) in the accretion-powered emission from many of these sources (see, e.g., van der Klis 2000 for a review). These oscillations have high frequencies (up to $\sim 1300 \mathrm{~Hz}$ [van Straaten et al. 2000], the highest-frequency astrophysical oscillations ever observed), high amplitudes (up to $15 \% \mathrm{rms}$ in the $2-60 \mathrm{keV}$ band of the Proportional Counter Array on $R X T E$ ), and high coherences (with quality factors $Q \equiv \nu / \mathrm{FWHM}>100$ in many cases), and often appear as two (but no more) high-frequency oscillations in a single power density spectrum. Beat-frequency models (BFMs) were quickly proposed for this phenomenon (Strohmayer et al. 1996; Miller, Lamb, \& Psaltis 1998). In these models, the higher-frequency of the two oscillations is attributed to the orbital frequency of gas at some special radius near the star, and the lower-frequency oscillation is a beat between this orbital frequency and the stellar spin frequency. These models are consistent with many of the trends evident in the early data, including the approximate constancy of the frequency difference between the two simultaneous kilohertz oscillations and the close match in four sources of this frequency difference with the stellar spin frequency inferred from brightness oscillations during thermonuclear X-ray bursts.

It has, however, been established recently that in several sources the frequency difference is not constant, and indeed can vary by more than $50 \mathrm{~Hz}$. Moreover, this variation is systematic: the higher the lower peak frequency, the lower the frequency difference. The explicability of this behavior in the beat-frequency picture has direct 
bearing on some of the most important inferences drawn from the kilohertz QPOs. For example, only in BFMs is the leveling-off of the frequency of both kilohertz QPOs possibly observed from $4 \mathrm{U} 1820-30$ (Zhang et al. 1998) a signature of the presence of the innermost stable circular orbit (ISCO), a crucial prediction of stronggravity general relativity. Hence only in BFMs can one infer a high gravitational mass $M>2.1 M_{\odot}$ for this source, which constrains strongly the equation of state of the high-density matter in the core of neutron stars.

The changing separation frequency observed in several sources provided part of the motivation for the development of other models of the kilohertz QPOs, in particular the relativistic precession models (e.g., Stella \& Vietri 1998). In these models the close match between the separation frequency and the spin frequency inferred from burst brightness oscillations is a coincidence, but they do predict the qualitative effect of a separation frequency that drops with increasing kilohertz QPO frequency.

Here we discuss the beat-frequency model in light of these new developments and contrast it with alternate pictures. In $\S 2$ we describe the observational trends that motivated the development of beat-frequency models. We then elaborate on these models, in particular the sonic-point beat-frequency model. In $\S 3$ we discuss the evidence for a changing difference frequency in the four sources Sco X-1, $4 \mathrm{U} 1608-52,4 \mathrm{U} 1728-34$, and $4 \mathrm{U} 1735-44$. We show that an aspect of the sonic-point beat-frequency model, included in the dynamics but originally omitted from the frequency estimates, naturally accommodates the changing difference frequency and can quantitatively fit the data. Finally, in $\S 4$ we contrast some of the predictions of the beat-frequency model with the predictions of the relativistic precession model, and discuss analysis that might be done with current data to help discriminate between the two interpretations. We also explore the impact of future observations, both with the upcoming generation of high spectral resolution satellites (such as Chandra and XMM) and with longer-term projects such as Constellation-X and a hypothetical high-area follow-on to $R X T E$.

\section{MOTIVATION FOR BEAT-FREQUENCY MODELS}

As discussed in the introduction, soon after the discovery of kilohertz QPOs it was established that these oscillations have (1) high frequency, (2) high amplitude, and (3) high coherence, and that there are always two or fewer kilohertz QPOs in a given power density spectrum. In addition, the separation frequency appeared consistent with constant in many sources and close to the spin frequency inferred from burst brightness oscillations in the four sources where this could be tested. It is now known (see $\S 3$ ) that in several, and perhaps all, sources, the separation frequency is not constant (although it is still close to the inferred spin frequency), and in fact decreases systematically with increasing lower peak frequency. In $\S 3$ we discuss how this new result may be interpreted within the beat-frequency model.

The high frequency indicates that the source of the brightness oscillations is close to the neutron star. A natural candidate for these oscillations is the orbital 
frequency at some special radius. Given that the burst oscillation frequency is most convincingly interpreted as the stellar spin frequency or its first overtone (see, e.g., Strohmayer \& Markwardt 1999), the close match of the separation frequency with the inferred stellar spin frequency suggests a sideband relation between the two simultaneous kilohertz QPOs. The lack of a third kilohertz QPO suggests a beatfrequency relation, because most other mechanisms will produce both an upper sideband and a lower sideband. For example, amplitude modulation of one frequency by another will produce two sidebands of equal strength. For all of these reasons, beat-frequency mechanisms are natural to propose for the kilohertz QPO phenomenon.

The qualitative match of beat-frequency expectations with the data is not enough to accept this model: it is also important to establish a reason why a particular radius in the accretion disk would be selected. The high coherence of the oscillations requires, in this model, that the radial range from which the oscillations are generated has a fractional width less than $\sim 1 / Q$, or less than $\sim 1 \%$ in the most stringent cases. Moreover, the high amplitudes observed in some sources indicates that the luminosity cannot be generated in just the small range of radii where the frequency is determined.

For these reasons, the sonic-point beat-frequency model was proposed (Miller, Lamb, \& Psaltis 1998). In this model, the special radius at which the frequencies are generated is where the inward radial velocity of the gas in the disk increases rapidly with decreasing radius. As described in Miller, Lamb, \& Psaltis (1998), this rapid increase in inward radial velocity is usually caused by radiation drag, which removes angular momentum from the accreting gas and hence causes it to spiral inwards. If for some reason radiation drag is not effective in a particular instance, as might happen if the accretion rate is so high that the optical depth from the stellar surface is large, then a similar rapid increase in velocity will nonetheless occur near the ISCO, where the inspiral of gas will open up simply because of gravitational effects. The radial velocity typically goes from subsonic to supersonic in this transition, and it is therefore convenient to label it $r_{\text {sonic }}$, the sonic point. However, in this model it is only the rapid change in velocity, and not the specific fact that the gas crosses a sonic point, that is important.

In this model we assume that there are dense clumps of gas orbiting at many radii near the star, and as we show in Miller, Lamb, \& Psaltis (1998) only the clumps near $r_{\text {sonic }}$ will produce sharp brightness oscillations. As gas streams from the clump onto the star, it produces a bright pattern of impact that rotates with the clump (see Miller, Lamb, \& Psaltis 1998), and hence a distant observer sees a modulation of the flux from the system as the bright impact spot passes into and out of view. This occurs at approximately the orbital frequency of the clumps at $r_{\text {sonic }}$. If the intensity of radiation from the stellar surface is also modulated at the stellar spin frequency, as it will be if a weak stellar magnetic field funnels extra matter towards one or two magnetic poles, then this variation in intensity modifies the mass accretion rate from the clumps, and hence changes the total luminosity from the impact spots. As shown in Miller, Lamb, \& Psaltis (1998), this 
creates an observed luminosity modulation from the system at approximately the beat frequency between the orbital frequency of the clumps and the stellar spin frequency. In this model the luminosity in the oscillations is generated at the stellar surface, where most of the gravitational energy is released, and hence the oscillations can have high amplitudes.

We now demonstrate that in this model the slow inward drift of the clumps, included in the dynamical calculations but not the frequency estimates of Miller, Lamb, \& Psaltis (1998), generally produces a separation frequency between the pair of kilohertz QPO peaks that is less than the stellar spin frequency.

\section{CHANGING DIFFERENCE FREQUENCY}

\subsection{Observational Evidence}

By late 1996, observations of Sco X-1 by van der Klis and colleagues (van der Klis et al. 1997; see also Méndez \& van der Klis 2000) demonstrated that the separation frequency between the kilohertz QPO peaks in this source is definitely not constant, and in fact that the separation clearly decreases with increasing lower peak frequency. This is contrary to the simplest expectations of the beat frequency model, in which the clumps generating the frequency are orbiting at a constant radius and hence the separation is extremely close to the stellar spin frequency.

Many explanations for this behavior were discussed in the community. Most of them centered on the apparently unique nature of Sco X-1 in this respect and hinged on other of its properties such as its very high (near-Eddington) luminosity at the point when the change in the difference frequency was most pronounced. These explanations included jet models (van der Klis et al. 1997) and other unpublished ideas that involved effects such as the finite thickness of the accretion disk. These models all had different physical bases and even different mathematical models for the changing difference frequency, but were all able to fit the relatively smooth $\Delta \nu$ vs. $\nu_{\text {lower }}$ curve for Sco X-1.

In the last year or so, however, careful analyses by Méndez and colleagues have shown that Sco X-1 is not unique in having a changing difference frequency. Specifically, the low-luminosity sources $4 \mathrm{U}$ 1608-52 (Méndez et al. 1998), 4U 1728-34 (Méndez \& van der Klis 1999), and 4U 1735-44 (Ford et al. 1998) also have difference frequencies that drop with increasing lower peak frequency, and the separation frequency in $4 \mathrm{U} 1636-536$ is slightly but significantly less than the spin frequency inferred from burst brightness oscillations (Méndez, van der Klis, \& van Paradijs 1998). More generally, Psaltis et al. (1998) showed that, within the observational uncertainties, all of the kilohertz QPO sources have separation frequencies that are consistent with this trend. This is therefore clearly not an effect that requires a high luminosity, and more general explanations must be sought. 


\subsection{Explanation Within the Beat-Frequency Model}

As described in detail in Lamb \& Miller (2000), and as we now discuss, in the sonicpoint model the inward drift of the clumps tends to increase the frequency of the lower kilohertz peak and decrease the frequency of the upper kilohertz peak. This produces a difference frequency that is generally less than the stellar spin frequency, in agreement with observations.

If a clump were to orbit in a perfect circle, then the inspiral time and phase traversed during inspiral of gas from the clump to the stellar surface would be the same regardless of when the gas separated from the clump. In this case, consider two successive "beats", i.e., two successive maxima of the mass flow rate from the clump. These occur a time $\Delta T=1 /\left(\nu_{\text {orb }}-\nu_{\text {spin }}\right)$ apart. Because the inspiral time to the surface is the same for each of the two beats, the arrival time at the surface is separated by exactly that same $\Delta T$, and the observed frequency of the beat is therefore just $1 / \Delta T=\nu_{\text {orb }}-\nu_{\text {spin }}$.

Now consider clumps that are drifting in slowly. Then the inspiral time decreases as time progresses. If we again think of two successive beats, suppose that the inspiral time for the second beat is a small time $\delta t$ less than the inspiral time for the first beat. Then the interval between releases of gas from the clump in the first and second beats is still $\Delta T=1 /\left(\nu_{\text {orb }}-\nu_{\text {spin }}\right)$, but the arrival times at the surface are now separated by $\Delta T-\delta t$. Therefore the observed frequency, which depends on surface arrival times because the surface is where the luminosity is generated, is $1 /(\Delta T-\delta t)$, which is higher than it would have been without the inward drift. This is somewhat analogous to Doppler blueshifting. Further analysis and comparison with numerical simulations shows (Lamb \& Miller 2000) that the upper peak frequency is also affected. In fact, the inward drift decreases the upper peak frequency, typically by a fractional amount that is $\sim 50 \%$ of the fractional amount by which the lower peak frequency is increased compared to the circular orbit approximation. Both the increase in the lower peak frequency and the decrease in the upper peak frequency act to decrease the frequency separation.

As described in Lamb \& Miller (2000), the magnitude of the change in the frequencies depends on the details of the inspiral, and in particular on how the total phase and time of the inspiral depend on the radial location of the clump. However, much of the physics is captured in a simple model in which the frequency change depends only on the ratio of two radial velocities: the velocity $v_{\text {clump }}$ of the clump near the sonic point and a characteristic velocity $v_{\text {gas }}$ of the gas when it leaves the clump (see Lamb \& Miller 2000 for more details). The observed frequencies are then approximately $\nu_{\text {low }}=\nu_{\text {beat }} /\left(1-v_{\text {clump }} / v_{\text {gas }}\right)$ and $\nu_{\text {high }}=\nu_{\text {orb }}\left(1-\frac{1}{2} v_{\text {clump }} / v_{\text {gas }}\right)$, where $\nu_{\text {beat }} \equiv \nu_{\text {orb }}-\nu_{\text {spin }}$ and $\nu_{\text {orb }}$ is the orbital frequency at the sonic point. Numerical simulations show that the characteristic velocity $v_{\text {gas }}$ of the gas is fairly insensitive to radius, and hence for simplicity we assume that $v_{\text {gas }}$ is independent of radius.

What remains is to produce a physical model for $v_{\text {clump }}$ as a function of radius. The radial velocity of a clump with angular momentum $J$ under the influence of a 
torque $N$ is $v_{r}=N /(\partial J / \partial r)$. In a Schwarzschild spacetime, for clumps whose mass does not depend on the distance from the star,

$$
\partial J / \partial r \propto \frac{1}{2} \frac{r-6 M}{(r-3 M)^{3 / 2}},
$$

where we use geometrized units in which $G \equiv c=1$. The singularity at $r=6 M$ in the equation for the radial velocity is only apparent. In reality, the velocity deviates from this simple formula near the ISCO. Numerical simulations show (Lamb \& Miller 2000) that for plausible torques the radial velocity of the clump can be small enough that even near the ISCO the inspiral time of the clumps is large, and hence the coherence of the brightness oscillations can be as high as observed. The simulations also show that for small enough torques the separation frequency can remain almost constant even near the ISCO. Therefore, in this model the observations of $4 \mathrm{U}$ 1820-30, which indicate an approximately constant separation frequency even when the kilohertz QPO frequencies may be leveling off, are still consistent with the asymptotic frequency being approximately the orbital frequency at the ISCO (Lamb \& Miller 2000).

As a particular model of the torque, assume that the clumps interact only weakly with each other and with any background gas in the accretion disk, so that the primary torque is exerted by the stellar magnetic field. The general form of the torque at radius $r$ is then $N \sim B^{2} A r$, where $A$ is the cross-sectional area of the clump and $B$ is the strength of the stellar magnetic field at the location of the clump. Assume also that the clump mass is approximately independent of the distance from the star, and that the clumps are in pressure equilibrium with the external magnetic field. Suppose that the clumps are kept at the local Compton temperature, which is roughly independent of the radius. Then $n k T \sim B^{2} \sim r^{-6}$ for a dipolar field, meaning that the diameter of the clumps scales as $r^{2}$ and the cross-sectional area scales as $r^{4}$. Then the torque scales as $N \sim r^{-1}$.

This model can then be fit to the data, using the relations between radial velocity and upper and lower QPO frequency given above. For a source such as Sco X-1 which has an unknown spin frequency, there are three parameters: the stellar gravitational mass $M$, the spin frequency $\nu_{\text {spin }}$, and the coefficient of the torque. For a source such as $4 \mathrm{U} 1728-34$ with a known $\nu_{\text {spin }}=364 \mathrm{~Hz}$, there are two free parameters. Figure 1 shows the results of the fitting. It is encouraging that one physical model can fit both of these sources as well as it does, especially given the high quality of the data for Sco X-1 (this model also fits $4 \mathrm{U}$ 1608-52 and 4U 1735-44).

\section{TESTS OF MODELS WITH CURRENT AND FUTURE DATA}

With the immense archive of data accumulated with $R X T E$, there are a number of further analyses that could in principle be performed to discriminate between models or test predictions of models. In this section we discuss such tests, partic-

ularly as they might discriminate between beat-frequency models and relativistic precession models, and also look to the future to consider the qualitatively new 


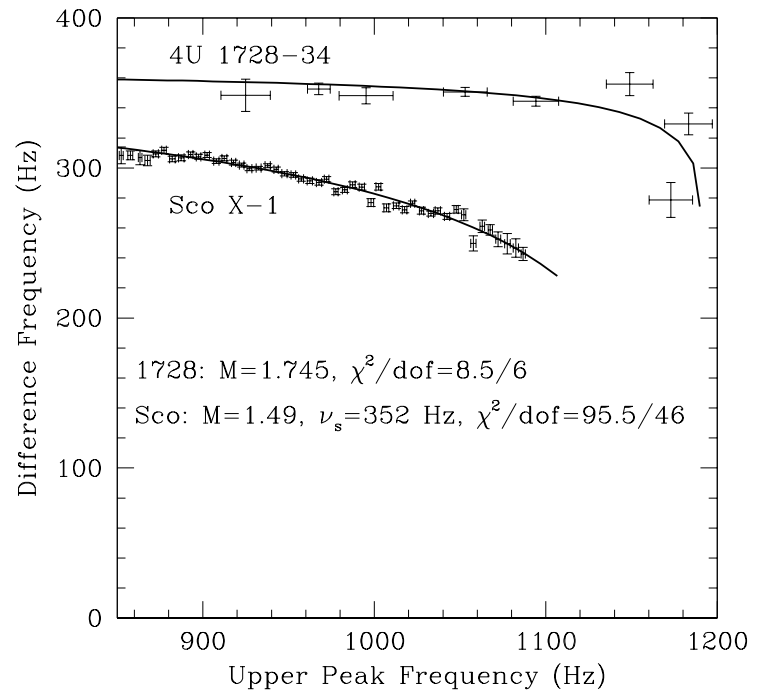

FIGURE 1. Difference frequency data and fit using the drifting clump model described in this section. The data for $4 \mathrm{U} 1728$ 34 and Sco X-1 were kindly provided by Mariano Méndez. The spin frequency for $4 \mathrm{U}$ 1728-34 was fixed at $364 \mathrm{~Hz}$ to correspond to the frequency of burst brightness oscillations in that source.

types of observations that may be available with the upcoming generation of X-ray satellites.

\subsection{Tests With Current Data}

An important difference between the predictions of the beat-frequency model and the relativistic precession model concerns expectations for the value of the stellar spin frequency. In the beat-frequency model it is expected that the stellar spin frequency is close to (although slightly larger than) the separation frequency, and is also very close to the burst oscillation frequency or half the burst frequency, depending on the symmetry. In contrast, as recently emphasized by Psaltis \& Norman (2000), in the simplest version of the relativistic precession model the data on the lower frequency horizontal branch type brightness oscillations combined with the kilohertz oscillations requires that the stellar spin frequency exceed $\sim 500 \mathrm{~Hz}$ in all sources, and be especially large (up to $\sim 900 \mathrm{~Hz}$ ) in the Z sources. Simple estimates of corrections due to fluid viscosity (Psaltis \& Norman 2000) suggest that these corrections would increase further the required spin frequency. The predictions for $\nu_{\text {spin }}$ therefore differ greatly in the two pictures, and if a clear spin frequency is detected in the persistent, accretion-powered emission then this will have strong bearing on the modeling. In addition, if it happens that the spin frequency is not equal to the burst oscillation frequency or half of it, this will overturn the apparently compelling arguments that burst brightness oscillations can only be explained via a rotational modulation mechanism. 
The two models also differ in their predictions of the frequencies of other, weak brightness oscillations. In the relativistic precession model, there is an upper sideband of the orbital frequency expected (Psaltis \& Norman 2000). The amplitude of this oscillation need not be as great as the amplitude at the lower sideband; for example, if the underlying perturbations of the accretion disk cut off sharply at frequencies exceeding the observed orbital frequency then the upper sideband could be weaker. Nonetheless, the oscillation is expected to be there at some level. In the beat frequency model there is an oscillation expected at twice the observed lower peak frequency. This is expected for several reasons. For example, the waveform generated by the beat is not expected to be perfectly sinusoidal, and hence overtones will be generated. In addition, if the radiation pattern rotating with the star, which helps produce the beat, is itself not perfectly sinusoidal, then it will generate weak overtones of the lower peak frequency.

The predicted frequencies discussed above depend only on the observed frequencies. If the observed frequencies are $\nu_{\text {low }}$ and $\nu_{\text {high }}$, then the predicted frequency in the relativistic precession model is $2 \nu_{\text {high }}-\nu_{\text {low }}$ and the predicted frequency in the beat frequency model is $2 \nu_{\text {low }}$. The frequencies will change with time for a given source, but these predictions are ideally suited for testing by use of the shift-and-add method first used by Méndez et al. (1998) to discover a weak brightness oscillation in $4 \mathrm{U} 1608-52$.

\subsection{Tests With Future Data and Satellites}

The launch of a new generation of high spectral resolution satellites such as Chandra and XMM opens up new ways to probe the strong gravity and dense matter of neutron stars. For example, suppose that simultaneous observations of a kilohertz QPO source are performed with $R X T E$ and XMM. If an Fe K $\alpha$ profile from the inner edge of the nearly-circular flow is detected and characterized with XMM, this profile gives the radius of that inner edge in units of the gravitational mass of the neutron star. If at the same time a pair of kilohertz QPOs is detected with RXTE, then the frequency of the upper peak, combined with the radius in units of the gravitational mass, yields the mass of the star. This is true independent of where the inner edge is; that is, it need not be at the innermost stable circular orbit. Therefore, such simultaneous measurements could provide a clean way to measure the gravitational masses of neutron stars in low-mass X-ray binaries.

Future, high-area missions such as Constellation-X or a hypothetical high-area follow-up timing mission to $R X T E$ could provide even more information, by allowing characterization of the waveforms of the brightness oscillations seen in accretionpowered and burst-powered emission. An example is shown in the left panel of Figure 2, which shows theoretical waveforms for burst brightness oscillations. The curve for the more compact star is broader, as is expected due to the extra gravitational light deflection. In addition, the curve for the larger (less compact) star is more asymmetric, due to the greater Doppler shifts from the surface rotation velocity. The waveform therefore encodes information about both the mass and radius 

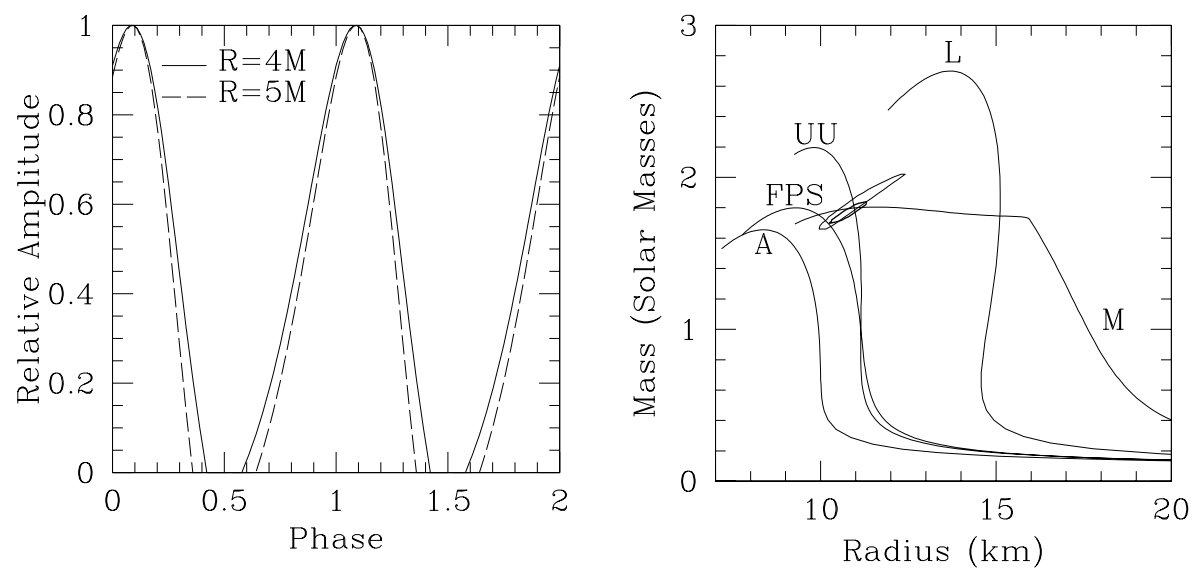

FIGURE 2. (left panel) Theoretical waveforms for burst brightness oscillations. Here we assume a small bright spot on the rotational equator, as seen by a distant observer in the rotational plane. We show simulated light curves over two cycles for a star with a gravitational mass $M=1.8 M_{\odot}$ and stellar radii $R=4 G M / c^{2}$ (solid curve) and $R=5 G M / c^{2}$ (dashed curve), with a stellar spin frequency as seen at infinity of $364 \mathrm{~Hz}$ (the spin frequency of $4 \mathrm{U}$ 1728-34, the source most frequently observed with $R X T E$ to have burst brightness oscillations). (right panel) Constraints on mass and radius possible with waveform fitting of burst brightness oscillations. We simulated the bolometric waveform from a $1.8 M_{\odot}$ star rotating at $364 \mathrm{~Hz}$, with high-density equation of state UU, a small emitting spot on the rotational equator, and no scattering after emission from the surface. We then performed a likelihood analysis using this simulated waveform. We combined the constraints from five bursts, assuming 5 seconds each of $5 \%$ rms amplitude, and a flux typical of the bursts observed with $R X T E$. The light outer contour shows the $1 \sigma$ confidence region expected from observations with Constellation-X, and the dark inner contour shows the $1 \sigma$ confidence region expected for a hypothetical future $10 \mathrm{~m}^{2}$ timing satellite. The light solid curves show the mass-radius relations given by different high-density equations of state, labeled as in Miller, Lamb, \& Psaltis (1998).

of the star, meaning that repeated observations of bursts from a single source can constrain the mass and radius tightly, with consequent constraints on the equation of state of the high-density matter in the core of neutron stars.

The right panel of Figure 2 shows an example of the constraints possible with Constellation-X (outer contour, at $1 \sigma$ ) and a hypothetical $10 \mathrm{~m}^{2}$ future timing instrument (inner contour, at $1 \sigma$ ). Clearly, waveform fitting can in principle yield very precise information about the mass and radius of individual neutron stars, and therefore about the equation of state of matter at supranuclear densities.

High-area timing missions also will potentially allow us to do qualitatively new types of tests of the strong-gravity predictions of general relativity. For example, we have just discussed two independent ways of estimating the gravitational mass of a neutron star: by combining Fe K $\alpha$ profiles with kilohertz QPOs, and by wave- 
form fitting of burst brightness oscillations. If either of these is successful for an individual source, then we can predict precisely the orbital frequency at the innermost stable circular orbit. With high-area timing instruments we expect to see more cases like $4 \mathrm{U}$ 1820-30, in which there is leveling-off of the QPO frequency and thus evidence for the ISCO. The match of this asymptotic frequency with the frequency predicted from the gravitational mass and general relativity will provide us with unprecedented quantitative tests of general relativity in strong gravity. In conclusion, therefore, the continued qualitative and quantitative agreement of the beat-frequency model with observations of kilohertz QPOs has not only yielded important new constraints on the equation of state of the dense matter in the core of neutron stars and, possibly, the first direct evidence for unstable orbits around neutron stars, a key prediction of general relativity. It also indicates strongly that future observations of these sources, especially with high-area timing missions, will allow us to continue to make qualitative leaps in our observational understanding of strong gravity and dense matter.

\section{ACKNOWLEDGEMENTS}

We thank Michiel van der Klis and Tod Strohmayer for discussions about possible information from high-area timing missions. This research was supported in part by NASA ATP grant number NRA-98-03-ATP-028.

\section{REFERENCES}

Ford, E. C., van der Klis, M., van Paradijs, J., Méndez, M., Wijnands, R. A. D., \& Kaaret, P. 1998, ApJ, 508, L155

Lamb, F. K., \& Miller, M. C. 2000, in preparation

Méndez, M., \& van der Klis, M. 1999, ApJ, 517, L71

Méndez, M., \& van der Klis, M. 2000, MNRAS, in press astro-ph/0006243)

Méndez, M., van der Klis, M., \& van Paradijs, J. 1998, ApJ, 506, L117

Méndez, M., van der Klis, M., Wijnands, R. A. D., Ford, E. C., van Paradijs, J., \& Vaughan, B. A. 1998, ApJ, 505, L23

Miller, M. C., Lamb, F. K., \& Psaltis, D. 1998, ApJ, 508, 791

Psaltis, D. et al. 1998, ApJ, 501, L95

Psaltis, D., \& Norman, C. 2000, ApJ, submitted astro-ph/0001391)

Stella, L. \& Vietri, M. 1998, ApJ, 492, L59

Strohmayer, T. E., \& Markwardt, C. B. 1999, ApJ, 516, L81

Strohmayer, T. E., Zhang, W., Swank, J. H., Smale, A., Titarchuk, L., \& Day, C. 1996, ApJ, 469, L9

van der Klis, M. 2000, ARA\&A, in press astro-ph/0001167

van der Klis, M., Wijnands, R. A. D., Horne, K., \& Chen, W. 1997, ApJ, 481, L97

van Straaten, S., Ford, E. C., van der Klis, M., Méndez, M., \& Kaaret, P. 2000, ApJ, submitted astro-ph/0001480

Zhang, W., Smale, A. P., Strohmayer, T. E., \& Swank, J. H. 1998, ApJ, 500, L171 